\title{
COVID-19 PATIENTS ANALYSIS AND RISK PREDICTION BASED ON LIFESTYLE DISEASES THROUGH INDIAN DATASET
}

\author{
Vijaykumar Patil \\ Research Scholar at Department of \\ Computer Engineering, \\ Bharati Vidyapeeth College of \\ Engineering \\ Navi Mumbai, Maharashtra, India
}

\author{
Dr. Dayanand Ingle \\ Professor and Head at Department of \\ Computer Engineering, \\ Bharati Vidyapeeth College of \\ Engineering \\ Navi Mumbai, Maharashtra, India
}

\begin{abstract}
Abstrac - A Novel Coronavirus disease (COVID-19) is a transferable virus triggered by a recently revealed coronavirus. World Health Organization (WHO) declared it as pandemic worldwide. COVID-19 was originated from Wuhan, a city of China and spared over the more than 190 countries over the word. The USA, Spain, Italy, France even India and every country suffered a lot by this epidemic. The indications of COVID-19 are Fever, Cough, Shortness of breath or trouble in breathing, Chills, Repeated shaking with chills, Muscle torment, Headache, Sore throat which is normal as any formal flue which each individual feel during season transition. In this article, the statistical analysis like chi-square analysis, age-wise and diseases-wise classification of recovered and deceased patients are performed and also the different types of Machine Learning models like Multiple linear regression, Naive Bayes Classifier, and Multilayer Perceptron Classifier are proposed for formal analysis and risk prediction of patients with different age group and individuals having lifestyle-based diseases with COVID-19. The dataset used for this study downloaded from covid19india.org, available in .csv format which included travel history of patients, relation with any existing COVID-19 patient, and record of any lifestylebased diseases like diabetes, hypertension, respiratory problem, etc.
\end{abstract}

Keywords - COVID-19, Machine Learning, Multilayer Perceptron, Naïve Bayes

\section{INTRODUCTION}

Now-a-days diabetes, hypertension and respiratory problem are the types of disease are become worldwide problem wherein it starts with any stage of life. These types of diseases are becoming day by day serious issue that among two, every second individual is suffering from it. Their always exists a situation where we find many uncertainties and differences in opinions in medical diagnosis process, so they exist a need to find ultimate solution to avoid risk factor in life [9, 10]. COVID illness 2019 (COVID-19), brought about by serious intense respiratory disorder COVID 2 (SARS-CoV-2), is a continuous pandemic. The figure of individuals tainted by the Coronavirus infection is expanding quickly. Up to May 12, 2020, 1,436,198 instances of COVID-19 have been accounted for in more than 200 nations and regions, bringing about around 85,521 passing's (with a lethal pace of 5.95\%) [1]. This has coordinated to-wards the public health concern in the international local area, as the World Health Organization (WHO) recognized the episode to be a Public Health Emergency of International Concern (PHEIC) on 30th jan, 2020, and remembered it as a pandemic on March 11, 2020 [2, 3]. COVID-19 has influenced everybody is day by day lives. Something like 316 million individuals from every country have been approached to remain at home to hinder the pandemic. In this perspective, organizations have been helpless to make considerable changes. Working environment tasks of numerous organizations went virtual. The impact of the advanced change on efficiency and corporate culture has been concentrated widely. In the interim, what COVID-19 has meant for purchasers, and the utilization culture has gotten generally restricted consideration.

Numerous representatives have rushed to change in accordance with the computerized change. Online video gathering software Zoom reports a $78 \%$ development in profits, and Google Meet reports a roughly $60 \%$ increment in client deals, where individuals go through 2 billion minutes in online gatherings each day [4-5]. Employees might not have any desire to return to actual offices even after the pandemic facilitates. Administrators foresee that a portion of the effects from COVID 19 on work environments may be enduring, where adaptable work hours and computerized gatherings are relied upon to progressively supplant the conventional 


\section{International Journal of Engineering Applied Sciences and Technology, 2021 Vol. 6, Issue 5, ISSN No. 2455-2143, Pages 198-206 \\ Published Online September 2021 in IJEAST (http://www.ijeast.com)}

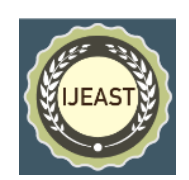

corporate culture [6]. Organizations are trying different things with decentralized dynamic and new software to make the new computerized work culture successful and as useful as working in actual offices [4]. Despite the fact that it is basic for organizations to make this change as successfully and quickly as could be expected, some different results that are basic to the reasonability of busi-nesses have often been forgotten about from the conversation - the effects of COVID 19 on purchasers and the market. Pandemic, even though it was viewed as an improbable occasion for quite a while before the COVID-19 episode, has been distinguished as one of the critical dangers to organizations, as indicated by a review led in 2007[12]. Virtual gatherings and task the board advancements have been progressively carried out even before the pandemic, and the computerized change has been moderately bothering free for some organizations [4]. The [7] pandemic as a gas pedal of the primary change in utilization and the computerized change in the commercial center. An audit coordinated in 1998 when e-straight introduced the better methodology for shopping on the web, tracked down that $46 \%$ of early adopters were using online business a significant part of the time, while simply $8 \%$ by and large adopters had an experience of web shopping [13, 14]. Nonetheless, the continuous examination on electronic shopping conduct exhibits that dis-semination may happen in light of framework impacts, yet the pandemic can moreover be a trigger. As shown by a continuous audit of 2,200 adults in the U.S., $37 \%$ of study respondents have considered moving to web shopping after COVID-19[15].

"Machine learning is getting popular in all industries with the main purpose of improving revenue and decreasing costs; by using Machine learning technique they automate and optimize their process to solve challenging tasks very efficiently $[19,20]$ ". Due to the reputation of ML in all the variety of applications and knowledge extraction, this research aims to broadly debate the role of lifestyle of an individual, age and various disease analysis are helpful to fighting the COVID-19 as well as in such future pandemic, which will rouse future pragmatic applications and methodological exploration[21]. In the accompanying investigation, first download information from covid19india.org for COVID-19 examination, and afterward pre-measure it as indicated by singular patient's outline measurement then, at that point sum up famous AI strategies in the expectation and information exporting. Finally, paper discuss several results of such statistical and machine learning analysis which helps to predict risk analysis of patients based on its lifestyle related diseases. Note that this study is mostly related dataset of COVID-19 studies up to April 29, 2020.

The rest of the paper is organized or structured as, section II is the Litrature Review which is the key works dealing with COVID 19 disease assessment, in Sect. III is designed as Proposed Methodologies used for the analysis of disease with co-morbidity. The dataset preparation and it attributes are illustrated in sect.-IV, the Result and discussion expounded in Sect. V. and definitive last section is conclusions are drawn in Sect. VI.

\section{LITERATURE REVIEW}

COVID-19, including picture securing, division, finding, and development. They especially center around the incorporation of X-beam and CT processed using AI, the 2 of which are broadly utilized in the forefront clinics, to delineate the most recent advancement of clinical imaging and radiology battling against COVID-19. Contrasted with the customary imaging work process that intensely depends on human works, AI empowers progressively sheltered, exact and effective imaging arrangements. The applications those late AI-engaged in COVID-19 principally incorporate the committed imaging stage, the contamination and lung and area division, the clinical appraisal and analysis, just as the spearheading fundamental and clinical research [8]. Additionally, numerous business items have been created, which effectively incorporate AI to battle COVID-19 and obviously exhibit the capacity of the innovation.

$N$. Zheng et al., in [5] cross breed man-made consciousness builds an AImodel is projected for COVID-19 prediction. To start with, as conventional pestilence models treat all people with coronavirus as experiencing a similar disease rate, an improved powerless contaminated model is intended to appraise the assortment of the disease rates for the diffusion laws breaking down and advancement pattern. Next, bearing in mind the impacts of control measures, avoidance and the expansion of the open's anticipation mindfulness, the new branch of NLP segment and the extensive memory is used to organize are implanted into the IISI model to assemble the half breed. The impact of government control gauges to be considered, the media's forthright statements, and the expansion in open mindfulness involving plague avoidance, this article utilizes ppretrained NLP models to remove highlights from applicable updates on different regions and urban areas. The LSTM is designed with extricated highlights are in this way joined with the system to address the variation of this disease rate evaluated by the IISI model, majorly which 
could predict the quantity of contaminated cases dependent different advancement pattern and the associating laws.

S. Park et al., in [10] proposed a motivated tactic by which the factual assessment of the CXR radiographs which having potential evidence for prediction. Exploratory outcomes of these methods used clinically understandable strikingness maps which show that this strategy achieves cutting edge implementation which is beneficial for COVID-19 decision. In this paper authors mean to additionally explore profound cnn and assess its plausibility for COVID-19 analysis. Tragically, under the present general wellbeing crisis, it is hard to gather huge arrangement of well-curated information for preparing neural network-based system. Hence, one of the fundamental focal points of this paper is to build up a neural system engineering that is reasonable for preparing with constrained preparing informational collection, which can at present produce radiologically interpretable outcomes. Since most as often as possible watched circulation examples of COVID-19 in CXR are respective inclusion, fringe dissemination and ground-glass opacification (GGO), an appropriately planned neural system ought to reflect such radiological discoveries. Besides, by broadening the possibility of the angle weighted class enactment map (Grad-CAM), which is designed based on novel probabilistic theory which is one more significant commitment of this paper that considers of fix insightful ailment likelihood in producing worldwide strikingness map. The subsequent class initiation map unmistakably shows the easily understandable outcomes that are all around connected with NN discoveries.

J. Zhang et al., in [11] proposed the Leverage advances, for example, built polymerases, isothermal intensification, and direct enhancement from complex grids may improve the profitability of current foundation, while rising advances like CRISPR diagnostics, pictorial end point recognition, and ePCR strategies for corrosive but nucleic detecting that may take as prompt o the at-home tests. The exercises scholarly, and advancements prodded from the COVID-19 pandemic could update our worldwide general wellbeing framework to more likely battle possible flare-ups later. The capacity to perform indicative examines inside each family unit can possibly upset in this current situation administration. They additionally checked on advances that can possibly bring athome sub-atomic tests to the real world. Bottlenecks in every innovation are recognized to empower future designing endeavors.

Theo Wibisono et al., in [12] introduced models to a great extent depend on homogeneous blending models, which treat everyone from the populace as having indistinguishable contamination hazard. Instinctively, such a supposition that is unreasonable. Certain segment gatherings (e.g., social insurance laborers, kids and the older), have higher disease dangers. Furthermore, personal conduct standards, for example, utilization of open transportation sway disease dangers. Utilizing contact systems to speak to the degree of contact between populace individuals and statistics information to rough geographic area and travel designs, they reproduce the movement of a bead spread sickness from the different areas under the Greter Toront. The outcomes are occasionally shown on maps of territory utilizing GIS for representation and arranging purposes.

\section{MATERIAL AND METHODOLOGY}

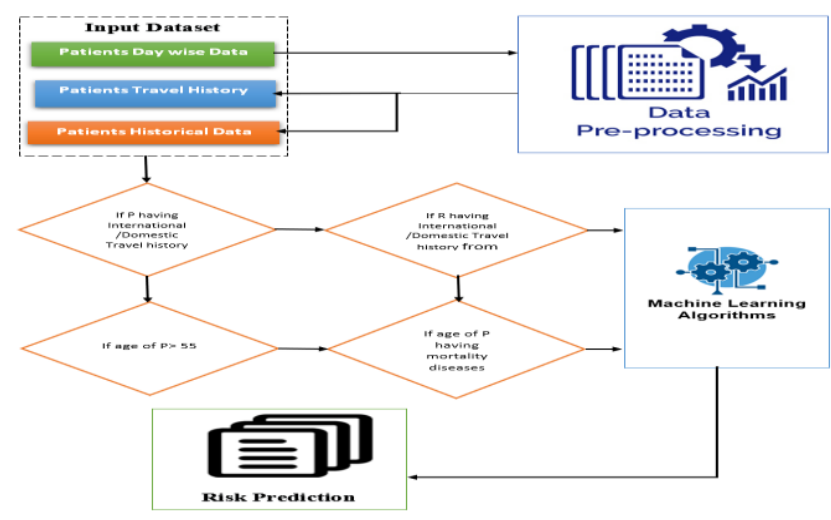

Fig. 1. Proposed Framework

The proposed methodology shown in Fig 1, which divided in three stages, first is to collecting dataset and perform preprocessing where only required patient's data is kept and other data (or attributes or tuples having in complete entries) which are not considered in proposed research are discarded. In proposed methodology patient referred as $\mathrm{P}$ and relative of referred as R. After finalization of data divided in three cases 1. Patients having travel history (Considered International/Domestic) including relative also, 2. Age wise categorization of data and 3 . The patients having pre-disease history like diabetes, heart and hypertension etc. Second statistical analysis performed on prepared dataset and then finally employed the Machine Learning technique to predict risk of COVID - 19. In proposed methodology the Chi square analysis, Other Statistical Measures (like age and disease wise classification), Multiple Regression Analysis, Multilayer Perceptron Analysis and Naive Bayes classification are applied to verify different factors which contribute in risk prediction of COVID-19 patients. 


\section{International Journal of Engineering Applied Sciences and Technology, 2021 \\ Vol. 6, Issue 5, ISSN No. 2455-2143, Pages 198-206 \\ Published Online September 2021 in IJEAST (http://www.ijeast.com)}

Multiple-linear regression (MLR): It also identified purely as statistical technique; a multiple regression forecast the outcome using numerous clarifying variables to of a response variable. The MLR designed by setting a only goal that is to model the association between the descriptive (independent) variables and reply to response (dependent) variable. The Formula for Multiple Linear Regression is

$$
\begin{aligned}
& y_{i}=\beta_{0}+\beta_{1} x_{i 1}+\beta_{2} x_{i 2}+\ldots+\beta_{p} x_{i p}+\epsilon \\
& \text { where, for } i=n \text { observations: } \\
& y_{i}=\text { dependent variable } \\
& x_{i}=\text { expanatory variables } \\
& \beta_{0}=\text { y-intercept (constant term) } \\
& \beta_{p}=\text { slope coefficients for each explanatory variable } \\
& \epsilon=\text { the model's error term (also known as the residuals) }
\end{aligned}
$$

The variables of dataset considered as follows to fit Multiple Linear Regression model

$$
\begin{aligned}
& \text { yi }=\text { dependent variable: risk ratio } \\
& \chi \mathrm{i} 1=\text { Travel history } \\
& \chi \text { i } 2=\text { Relative travel history } \\
& \chi \text { i } 3 \text { = Relative found COVID +ve } \\
& \chi \mathrm{i} 4=\text { Diabetes } \\
& \chi \mathrm{i} 5=\text { Heart disease } \\
& \chi \text { i } 6=\text { Brain issue } \\
& \chi \mathrm{i} 7=\mathrm{TB} \\
& \chi \text { i } 8=\text { Thyroid } \\
& \chi \text { i } 9=\text { Mental Disorder } \\
& \chi \mathrm{i} 10=\text { Respiratory problem } \\
& \chi \mathrm{i} 11=\text { Hypertension } \\
& \chi i 12=\text { Kidney } \\
& \chi i 13=\text { External injury } \\
& \beta 0=\text { age of an individual } \\
& \beta 1=\text { coefficient value of dependent variable } \\
& \text { when } \chi_{\mathrm{i} 1} \text { changes } \\
& \beta 2=\text { coefficient value of dependent variable } \\
& \text { when } \chi_{\mathrm{i} 2} \text { changes } \\
& \mathrm{C}=\text { the model error term }
\end{aligned}
$$

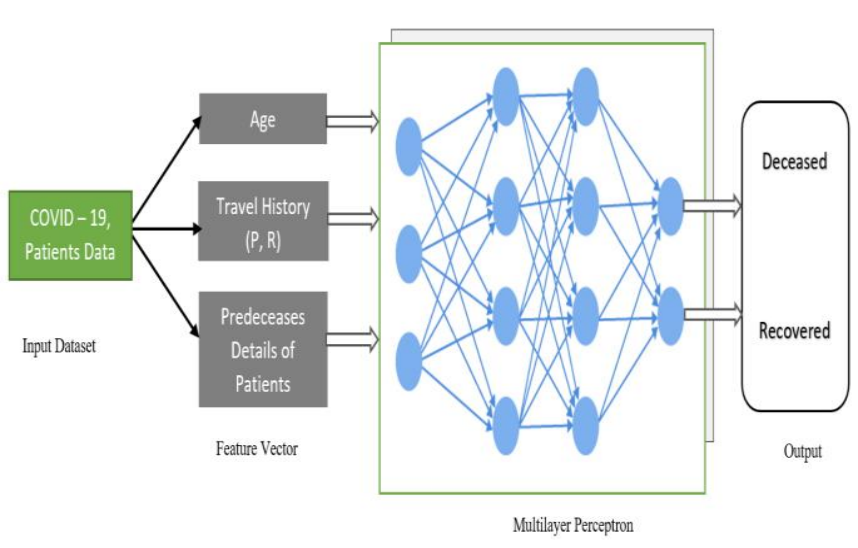

Fig 2. Proposed architecture of multilayer perceptron classifier.

Multilayer Perceptron Classification (MLP): It is a model designed based on ANN model which apply feed-forward maps to all possible sets of input data attributes onto a set of fitting output. The MLP designed with nodes having directed graph and multiple layers, with each layer from MLP is completely attached to the next layer. Aside from the info node, every node is a neuron (or handling component) with a non-direct actuation work [16]. The multilayer perceptron classifier architecture shown in Fig. 2. The fit the network model i.e. MLP with back propagation. The during design of current network model a Softmax function is applied as an activation function and Cross-entropy as an error function.

Backpropagation (Age, Travel History, Pre-decease details, $n, n$ in, nout, $n$ hidden)

The $i$ to $j$ is xij considered as input and the $i$ to unit $j$ is unit denoted wij called as weight from input unit.

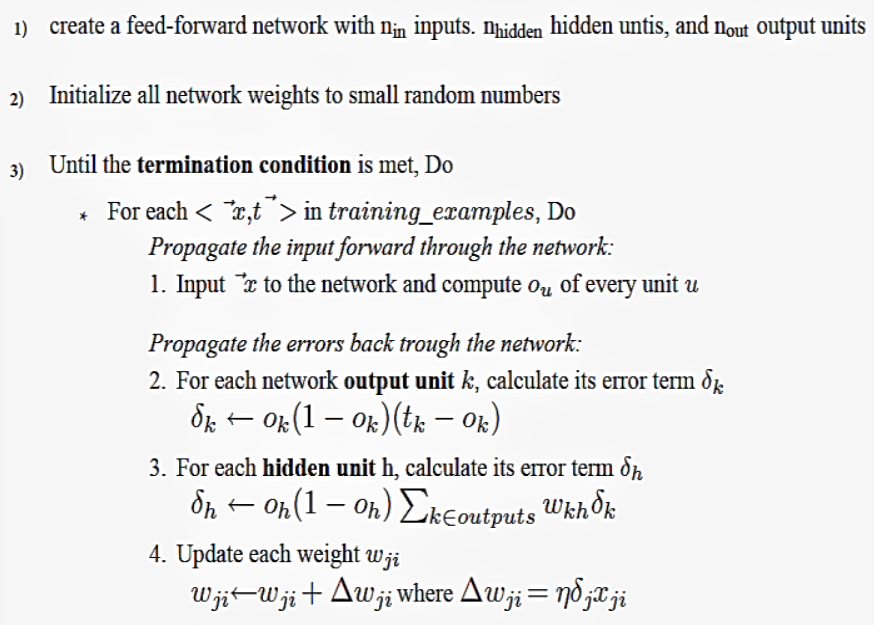

Fig 3. Minimize the errors through Back propagation algorithm [17]. The proposed method in his paper considered the subsequent closure circumstances in the MLP classifier: 


\section{International Journal of Engineering Applied Sciences and Technology, 2021 \\ Vol. 6, Issue 5, ISSN No. 2455-2143, Pages 198-206 \\ Published Online September 2021 in IJEAST (http://www.ijeast.com)}

- Fixed number of epochs or repetition.

- Threshold for error.

- Discrete validation set for rrror on a fall up to certain threshold.

Naive Bayes classifier: A NBC is a based on probabilistic approach which is popularly used for classification; it is implemented based on Bayes theorem shown as bellow.

$$
P(A \mid B)=\frac{P(B \mid A) P(A)}{P(B)}
$$

The hypothesis made here is that the predictors or features are shown in Table 1 all are impartial The age, travel history and predeceases data of patients used to training the Naive Bayes classifier model and corresponding target variable 'Patient_Status' (either Deceased or Recovered). To classify risk analysis of patient whether Deceased or Recovered based on age, travel history and predeceases. The above theorem rewritten as

$$
P(D \mid R)=\frac{(R 1 \mid D) P(D)}{P(R)}+\frac{(R 2 \mid D) P(D)}{P(R)}+\ldots+\frac{(R n \mid A D) P(D)}{P(R)} \ldots \ldots
$$

Were,

- $\mathrm{P}(\mathrm{D} \mid \mathrm{R})$ - probability (posterior) of deceased patients

- $\mathrm{P}(\mathrm{D})$ - prior probability of deceased patients.

- $\mathrm{P}(\mathrm{R} \mid \mathrm{D})$ - likelihood or probability of recovered patients or predictor

- $\mathrm{P}(\mathrm{R})$ - recovered predictors prior probability.

The measure the performance of different measured used evaluated using confusion matrix which is one of very wellknown measured in ML. The terms used here are TP, TN, FP and FN labeled below.

- TP (True positive): number of patients which actually deceased predicted by model.

- TN (True Negative): number of false prediction of patients which actually deceased predicted by model.

- FP (False positive): number of patients which actually deceased but predicted as recovered.

- FN (False Negative): number of false patients which actually deceased but predicted as recovered.

\section{DATASET PREPARATION}

The covid19.org provide different well-prepared dataset of COVID-19 patients from India, they also allow to use different forms of API to access it online and integrate in our application or for analysis. In this paper the file labeled as death_and_recovered.csv from covid-19 is used, it included attributes as follows

Table 1: Different attributes used to prepare Dataset

\begin{tabular}{|c|c|}
\hline Row Dataset Attributes & Attributes of Processed Dataset \\
\hline Date of admission & Age Bracket \\
\hline Age of Patient & Travel History of Patient \\
\hline Gender & Domestic travel History \\
\hline Current Status & Reason for travel \\
\hline City of Patient & Is relative +ve \\
\hline District & Is Patient Diabetes \\
\hline State & Any Heart disease \\
\hline State code & If Brain issues \\
\hline Notes (descriptive data) & Is Patient having TB \\
\hline Nationality of Patient & Is Patient having Thyroid \\
\hline & Is Mental disorder \\
\hline & Is Patient having Respiratory problem \\
\hline & Is Patient having Hypertension \\
\hline
\end{tabular}

The row dataset which is downloaded from covid-19.org contains "Notes" attribute. It contains data relevant to the covid patients in descriptive form which is about patents history which converted on categorical attributes as shown in Table 1. The processed dataset having 7408 rows in which only 433 patient's status is records and having a historical medical data is described as Note. The Status is categorical variable or predictor which having two binary values as first recovered which denoted as 0 and second one is deceased represented as 0 .

\section{RESUlT AND DisCUSSION}

\section{A. Chi-square analysis}

The analysis result of Chi square for finalized dataset shown as follows:

- Ha: The patients age is less than 55, having greeter percentage of Recovery

- H0: The patients age is less than 55, having less percentage of Recovery

Table 2: Age-wise Analysis of Deceased \& Recovered Patients

\begin{tabular}{|l|c|c|}
\hline \multicolumn{3}{|c|}{ Age-wise Analysis } \\
\hline & Age is $>=\mathbf{5 5}$ & Age is $<\mathbf{5 5}$ \\
\hline Deceased & 211 & 109 \\
\hline Recovered & 32 & 80 \\
\hline & 243 & 189 \\
\hline
\end{tabular}




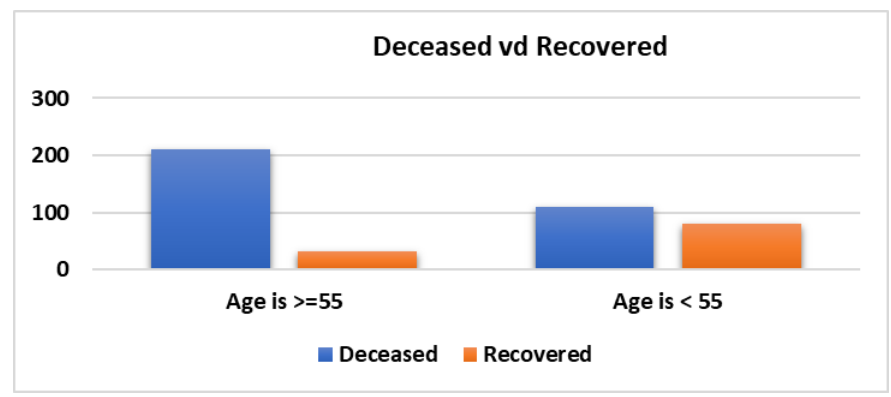

Fig 3: Age-wise Analysis of Deceased and Recovered Patients

Table 3: Chi-Square Test Statistics (Patients age >=55)

\begin{tabular}{lllll}
\hline & $\begin{array}{l}\text { Hypothesized } \\
\text { Proportion }\end{array}$ & Observed & Expected & $\begin{array}{l}\text { Chi-Square } \\
\text { Test }\end{array}$ \\
\hline Deceased & 0.65 & 211 & 157.95 & 17.81767965 \\
Recovered & 0.35 & 32 & 85.05 & 33.08997648 \\
& & 243 & & 50.90765613 \\
& & & p-value & $9.68152 \mathrm{E}-13$ \\
& & Test Statistic & 50.90773251 \\
\hline
\end{tabular}

Table 4: Chi-Square Test Statistics (Patient's age<55)

\begin{tabular}{lllll}
\hline & $\begin{array}{l}\text { Hypothesized } \\
\text { Proportion }\end{array}$ & Observed & Expected & $\begin{array}{l}\text { Chi-Square } \\
\text { Test }\end{array}$ \\
\hline Deceased & 0.65 & 109 & 122.85 & 1.561436711 \\
Recovered & 0.35 & 80 & 66.15 & 2.899811036 \\
& & 189 & & 4.461247747 \\
& & & p-value & 0.034672162 \\
& & & Test Statistic & 4.461247747 \\
\hline
\end{tabular}

The Table 2 and Fig 3 shows Age-wise Analysis of COVID - 19 patients which are categories as Deceased and Recovered with age as threshold value 55. This table shows 243 patients out of 432 having age is greater or equal to 55 and 189 patients age having less than 55. The Table 3 and Table 4 shows pvalue $9.68152 \mathrm{E}-13$ and 0.034672162 respectively. The p-value from Table 3 is far less than significance level 0.5 so it clearly indicates the null hypothesis are rejected strongly.

\section{B. Other Statistical Measures}

The age wise risk analysis concluded in Fig 4; it shows the no of patients (Frequency) vs age bracket Histogram with mean age 56 and std. dev 16, but it clearly indicates that the patients having age is greater than 55 having high risk of COVID -19. Fig 5 shows Nonlinear Regression: Exponential Model, here y is taking log of both sides shows relative intensity of age to the number of COVID-19 patients is 7.537e0.036x and R- Square indicate that $75.45 \%$ of variability of number of patients explained by variable age. Similarly, Fig 6 and Fig 7 shows age wise classification deceased and recovered patients, the relative intensity deceased patients is $2.2023 \mathrm{e} 0.05 \mathrm{x}$ and $\mathrm{R}$ - Square indicate that $80.40 \%$ of variability of number of patients those are deceased explained by variable age

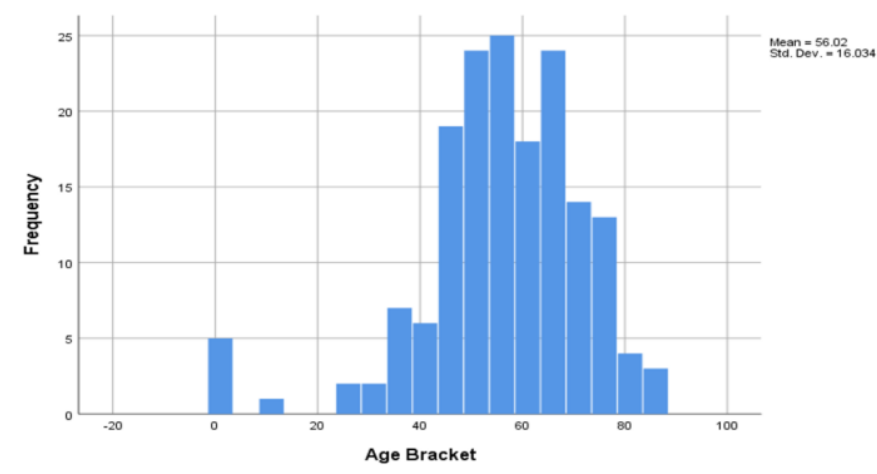

Fig 4: Histogram of COVID - 19 Patients

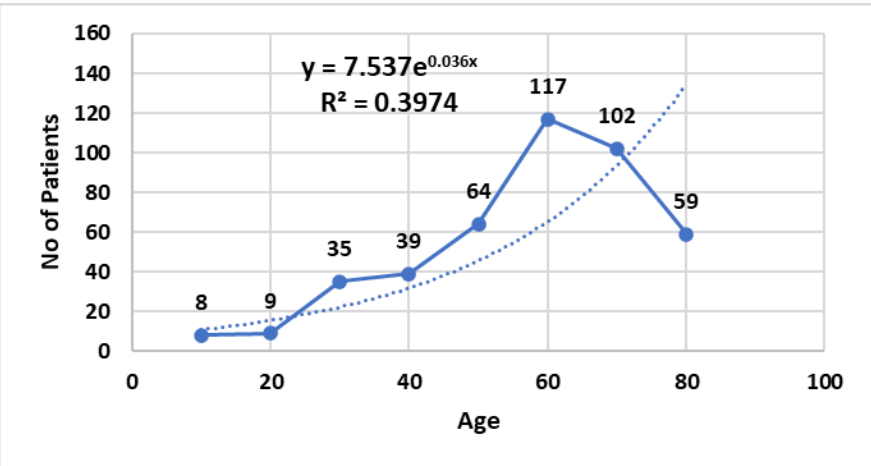

Fig 5: Age wise classification of all patients

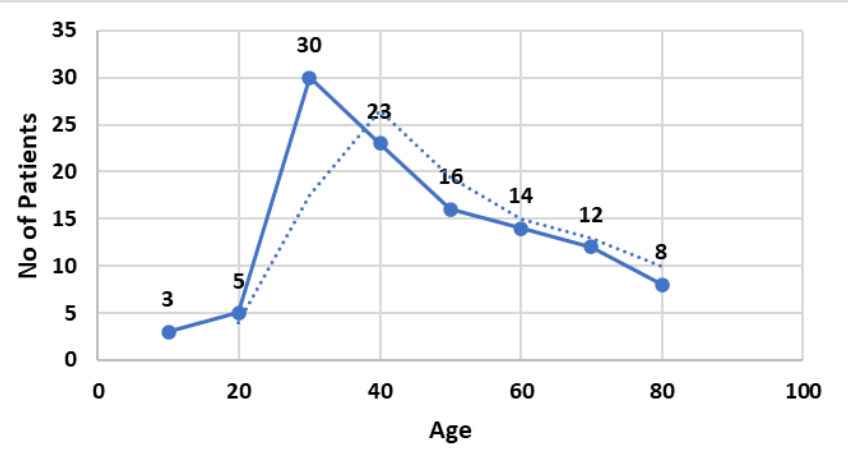

Fig 6: Age Wise Classification Recovered Patients

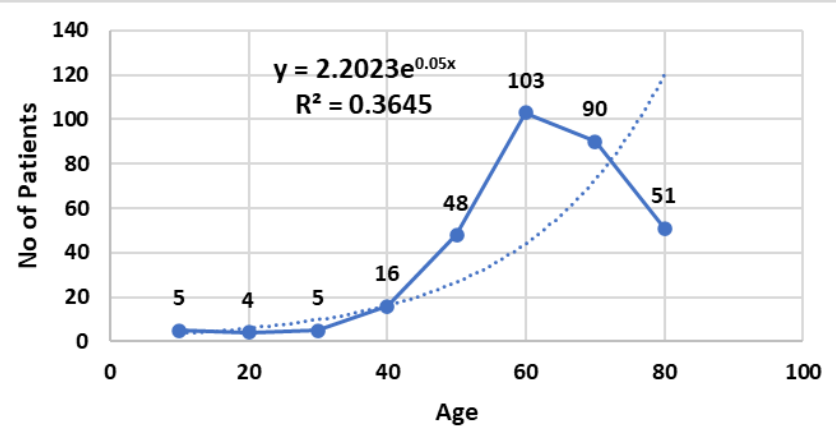

Fig 7: Age Wise Classification Deceased Patients 


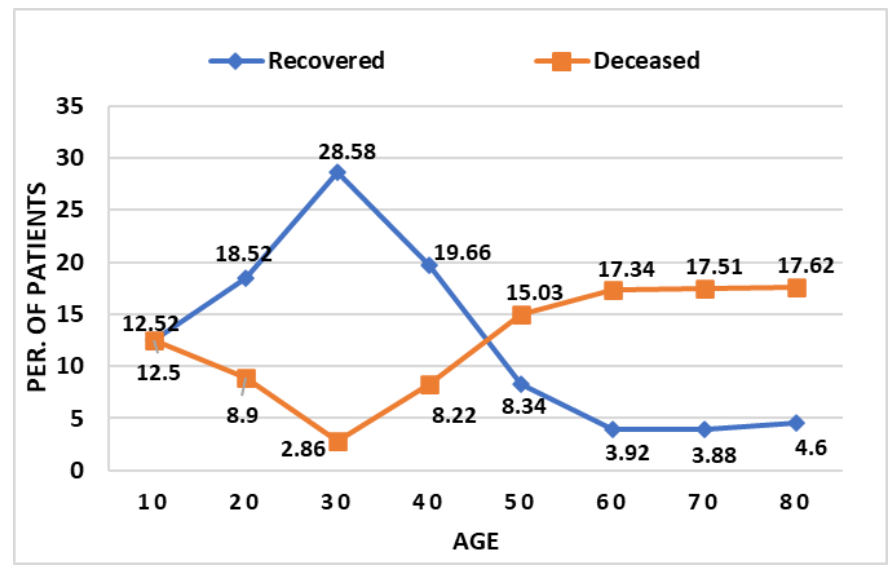

Fig 8: Recovered Vs Deceased

The Recovered Vs Deceased age wise risk analysis shown in Fig 8. It shows the patients average age between 28 to 30 having greater percentage of chance to recovery whereas age more than 56 may be the less. The Fig 9 shows the analysis of lifestyle-based diseases analysis of COVID-19 patients with different diseases like diabetics, heart, hypertension, kidney, respiratory problem, TB, thyroid, mental disorder etc. The individuals having diabetics, heart, hypertension and respiratory problem having high risk from COVID - 19 diseases than all other types of disease. These included diseases in risk analysis in Figure 8 are lifestyle disease which arises with age.

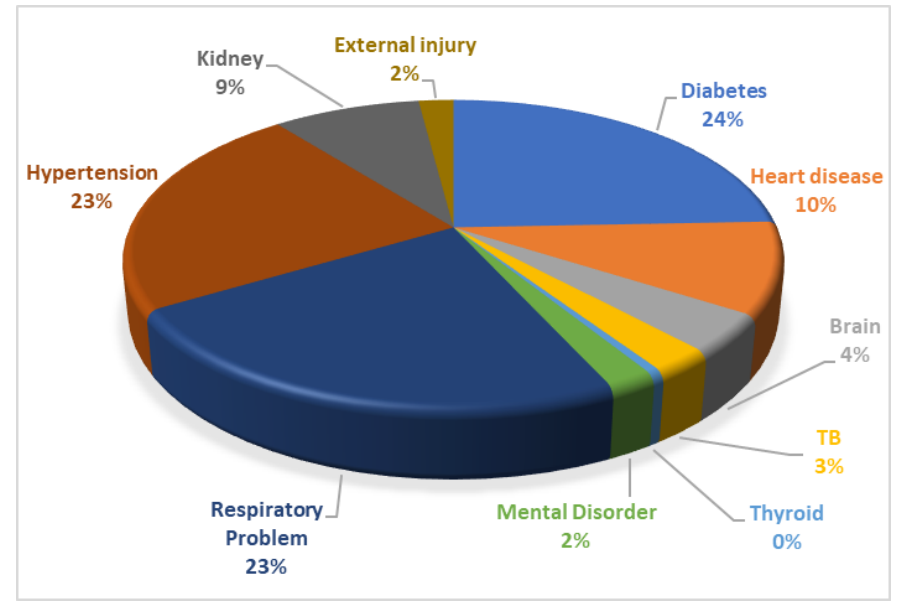

Fig 9: Disease wise Risk Classification of COVID-19 Patients

\section{Machine Learning Techniques}

In proposed methodology, Multiple Linear Regression, Naïve Bayes and Multilayer Perceptron (MLP) Classification are the types of Machine Learning techniques are used. Multiple Linear Regression and MLP are build using IBM SPSS Statistics tool. The Fig 10 shows Model Summary of Multiple Linear Regression; Here the Adjusted R Square values illustrate that the $42 \%$ variability of Patient_Status explained by Dependent variables (listed in Table 1). Fig 11 shows ANOVA test Table, it shows F-Value and P - Values as 14.329 and 0.00 respective which indicate strongly the null hypothesis 'The patients age is less than 55, having less percentage of Recovery' is rejected.

\begin{tabular}{ll|r|r|r} 
Model & $\mathrm{R}$ & R Square & \multicolumn{1}{c}{$\begin{array}{c}\text { Adjusted R } \\
\text { Square }\end{array}$} & $\begin{array}{l}\text { Std. Error of the } \\
\text { Estimate }\end{array}$ \\
\hline 1 & $.672^{\mathrm{a}}$ & .452 & .420 & .373 \\
\hline
\end{tabular}

a. Dependent Variable: Patient_Status

Fig 10: Summary of MLR

\begin{tabular}{|c|c|c|c|c|c|c|}
\hline Model & & $\begin{array}{l}\text { Sum of } \\
\text { Squares }\end{array}$ & df & Mean Square & $F$ & Sig. \\
\hline \multirow[t]{3}{*}{1} & Regression & 29.949 & 15 & 1.997 & 14.329 & $.000^{b}$ \\
\hline & Residual & 36.369 & 261 & .139 & & \\
\hline & Total & 66.318 & 276 & & & \\
\hline
\end{tabular}

a. Dependent Variable: Patient_Status

b. Predictors: (Constant), externalinjury, thyroid, mentaldisorder, TB, R_travel_History, F_travel_History, D_travel_History, kidney, hypertension, respiratoryproblem, heartdisease, ÄgeBracket, Diabetes, Brain, R_positive

Fig 11: ANOVA Table

The Model Summary of Multilayer Perceptron Classifier (Network) shown in Fig 12 and 13, which shows Cross Entropy Error and Percentage of Incorrect Predictions as 17.387 and $2.1 \%$ for training and 8.917 and $2.8 \%$ for testing respectively. The $73 \%$ of dataset used to train MLP and $27 \%$ records are to be used to train the model. The following Figure 12 shows table of Model Summary of MLP Classifier, during data preparation for MLP; the value 0 (zero) used to indicate patient status as Deceased and 1 (one) for Recovered patients. MLP result shows $97.2 \%$ of overall accuracy in the testing phase of model.

The machine learning classification model Naive Bayes build using python programming model where the $75 \%$ of dataset used to train and $25 \%$ are used test the model. The Table 4 shows Confusion Matrix generated through Naive Bayes classifier which shows statistics of actual vs predicted trade-off, where 32 observations are predicted as True Positive and 29 are False - Negative and Type-I error indicate 5 observations and 3 are from Type- II error. The accuracy of Naive Bayes classification model given as $88.41 \%$ and other statistical measures are shown in Table 5. 


\section{International Journal of Engineering Applied Sciences and Technology, 2021 \\ Vol. 6, Issue 5, ISSN No. 2455-2143, Pages 198-206 \\ Published Online September 2021 in IJEAST (http://www.ijeast.com)}

\begin{tabular}{llr} 
Training & Cross Entropy Error & 17.387 \\
\cline { 2 - 3 } & $\begin{array}{l}\text { Percent Incorrect } \\
\text { Predictions }\end{array}$ & $2.1 \%$ \\
\cline { 2 - 3 } & Stopping Rule Used & $\begin{array}{l}1 \text { consecutive } \\
\text { step(s) with no } \\
\text { decrease in } \\
\text { error }^{\mathrm{a}}\end{array}$ \\
\cline { 2 - 3 } & Training Time & \multicolumn{1}{c}{$0: 00: 00.10$} \\
\hline Testing & Cross Entropy Error & 8.917 \\
\cline { 2 - 3 } & $\begin{array}{l}\text { Percent Incorrect } \\
\text { Predictions }\end{array}$ & $2.8 \%$ \\
\hline
\end{tabular}

Dependent Variable: Patient_Status

a. Error computations are based on the testing sample.

Fig 12: Model Summary of Multilayer Perceptron Classifier

\begin{tabular}{ll|r|r|r} 
& & \multicolumn{4}{c}{ Predicted } \\
Sample & Observed & \multicolumn{1}{c}{0} & 1 & Percent Correct \\
\hline \multirow{2}{*}{ Training } & 0 & 188 & 0 & $100.0 \%$ \\
\cline { 2 - 5 } & 1 & 4 & 0 & $0.0 \%$ \\
\cline { 2 - 6 } & Overall Percent & $100.0 \%$ & $0.0 \%$ & $97.9 \%$ \\
\hline \multirow{2}{*}{ Testing } & 0 & 69 & 0 & $100.0 \%$ \\
\cline { 2 - 5 } & 1 & 2 & 0 & $0.0 \%$ \\
\cline { 2 - 5 } & Overall Percent & $100.0 \%$ & $0.0 \%$ & $97.2 \%$ \\
\hline
\end{tabular}

Dependent Variable: Patient_Status

Fig 13: Model Summary of Multilayer Perceptron Classifier Confusion matrix of the classifier

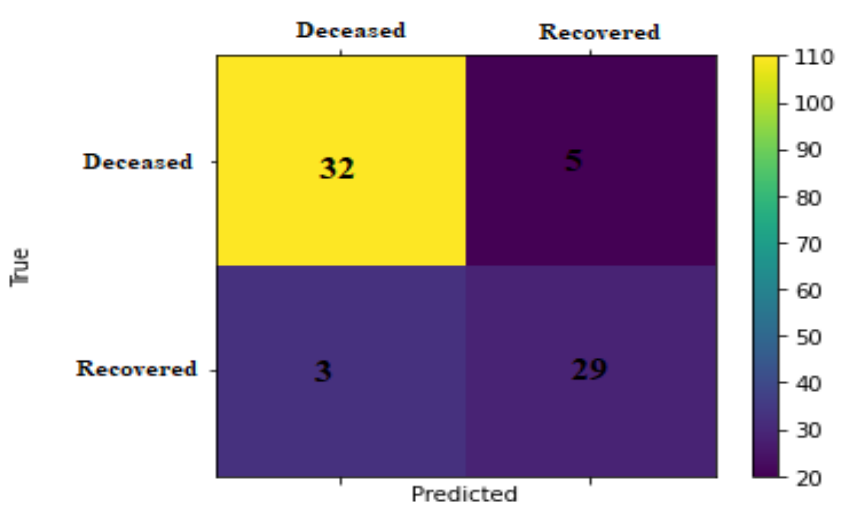

Fig 14: Confusion Matrix

Table 6: Result computation using Confusion Matrix

\begin{tabular}{|c|c|}
\hline Measure & Value \\
\hline Accuracy & $88.41 \%$ \\
\hline Misclassification & $11.59 \%$ \\
\hline Precision & $86.49 \%$ \\
\hline Sensitivity & $91.43 \%$ \\
\hline Specificity & $85.29 \%$ \\
\hline
\end{tabular}

\section{CONClusion}

In examining the proposed research so far, it has been seen that the risk of COVID - 19 patients is in more aged people, having age greater than 55 as well as the individuals those are already suffered from lifestyle-based diseases like diabetics, heart, hypertension, kidney, respiratory problem, mental disorder, etc. The proposed mechanism has been tested using statistical analysis (chi-square) and Machine learning algorithms like Linear and non-linear, Naive Bayes classification, and Multilayer Perceptron Classifier or Network. The statistical approach indicates that the variability of risk for COVID-19 disease explained by factors considered is in between $421 \%(\min )$ to $801 \%(\max )$. The prediction models also predict the risk with $881 \%$ ( $\min$ ) to $971 \%$ (max) accuracy level. The proposed study indicated the aged individual with already suffered from lifestyle-based diseases having a high risk of COVID-19. In future, accuracy level may be improved by adding more samples of data items and applying more powerful algorithms of machine learning or by increasing a greater number of hidden layers on MLP classifier. It will also improve by considering a greater number of diseases that individuals having because of their daily lifestyle. In the proposed study, only the Indian dataset is considered. In future, it may extend world-wide with a greater number of factors.

\section{REFERENCE}

[1] WHO. (April 10, 2020). Coronavirus disease 2019 (COVID-19) Situation Report - 80. Available: https://www.who.int/docs/default-

source/coronaviruse/situation-reports/20200409-sitrep-80covid-19.pdf?sfvrsn=1b685d64_4

[2] WHO. (2020, 30 January, 2020). Statement on the second meeting of the International Health Regulations (2005) Emergency Committee regarding the outbreak of novel coronavirus (2019-nCoV).

[3] WHO. (2020). WHO Director-General's opening remarks at the media briefing on COVID-19.

[4] F. Shi et al., "Review of Artificial Intelligence Techniques in Imaging Data Acquisition, Segmentation and Diagnosis for COVID-19," in IEEE Reviews in Biomedical Engineering, doi: 10.1109/RBME.2020.2987975.

[5] N. Zheng et al., "Predicting COVID-19 in China Using Hybrid AI Model," in IEEE Transactions on Cybernetics, doi: 10.1109/TCYB.2020.2990162.

[6] H. Kang et al., "Diagnosis of Coronavirus Disease 2019 (COVID-19) with Structured Latent Multi-View 
Representation Learning," in IEEE Transactions on Medical Imaging, doi: 10.1109/TMI.2020.2992546.

[7] R. Y. Kim, "The Impact of COVID-19 on Consumers: Preparing for Digital Sales," in IEEE Engineering Management Review, doi: 10.1109/EMR.2020.2990115.

[8] D. Dong et al., "The role of imaging in the detection and management of COVID-19: a review," in IEEE Reviews in Biomedical Engineering, doi: 10.1109/RBME.2020.2990959.

[9] R. F. Sear et al., "Quantifying COVID-19 content in the online health opinion war using machine learning," in IEEE Access, doi: 10.1109/ACCESS.2020.2993967.

[10] Y. Oh, S. Park and J. C. Ye, "Deep Learning COVID-19 Features on CXR using Limited Training Data Sets," in IEEE Transactions on Medical Imaging, doi: 10.1109/TMI.2020.2993291.

[11] J. Zhang et al., "Navigating the Pandemic Response Life Cycle: Molecular Diagnostics and Immunoassays in the Context of COVID-19 Management," in IEEE Reviews in Biomedical Engineering, doi: 10.1109/RBME.2020.2991444.

[12] T. Wibisono, D. M. Aleman and B. Schwartz, "A nonhomogeneous approach to simulating the spread of disease in a pandemic outbreak," 2008 Winter Simulation Conference, Miami, FL, USA, 2008, pp. 2941-2941, doi: 10.1109/WSC.2008.4736431.

[13] S. E. Pawar and S. S. Smita, "Study of diagnosis of diabetes mellitus under healthcare," 2016 3rd International Conference on Computing for Sustainable Global Development (INDIACom), New Delhi, 2016, pp. 2063-2066.

[14] D. Howland, The consumer after COVID-19, Retail Dive, April 8, 2020. [Online] https://www.retaildive.com/news/the-consumer-aftercovid-19/575634/

[15] Morning Consult, Crosstabulation Results, National Tracking Poll \#200394, March 26, 2020. [Online] https://morningconsult.com/wpcontent/uploads/2020/03/200394_crosstabs_CORONAVI RUS_CONTENT_Adults_v4_JB-1.pdf

[16] Spencer M, Eickholt J, Cheng J. A deep learning network approach to ab initio protein secondary structure prediction. IEEE Transactions on Comp Biol Bioinformatics 2014.

[17] Leo Dencelin X1, Ramkumar Analysis of multilayer perceptron machine learning approach in classifying protein secondary structures, Biomedical Research 2016; Special Issue: S166-S173

[18] Bengio Y. Practical recommendations for gradient-based training of deep architectures. Neur Netw Trade Spr 2012; 437-478.

[19] Patil, V., Ingle, D.R. An association between fingerprint patterns with blood group and lifestyle based diseases: a review. Artif Intell Rev (2020). https://doi.org/10.1007/s10462-020-09891-W

[20] P. N. Vijaykumar and D. R. Ingle, "A Novel Approach to Predict Blood Group using Fingerprint Map Reading," 2021 6th International Conference for Convergence in Technology (I2CT), 2021, pp. 1-7, doi: 10.1109/I2CT51068.2021.9418114.

[21] P. Vijaykumar and D. R. Ingle, " Comparative Analysis of Different ML Classification Algorithms with Diabetes Prediction through Pima Indian Diabetics Dataset," 2021 International Conference on Intelligent Technologies (CONIT) Karnataka, India. June 25-27, 2021 